\title{
A BAFF/APRIL-dependent TLR3-stimulated pathway enhances the capacity of rheumatoid synovial fibroblasts to induce AID expression and Ig class-switching in B cells
}

\author{
Bombardieri, M ; Kam, N W ; Brentano, F ; Choi, K ; Filer, A ; Kyburz, D ; McInnes, I B ; Gay, S ;
} Buckley, C ; Pitzalis, C

\begin{abstract}
OBJECTIVES: To dissect the role of toll-like receptor (TLR) signalling and B cell survival/proliferating factors in the crosstalk between rheumatoid arthritis synovial fibroblasts (RASF) and B cells. METHODS: RASF, rheumatoid arthritis dermal fibroblasts (RADF) and osteoarthritis synovial fibroblasts (OASF) were analysed for the expression of B cell survival/proliferating factors BAFF and APRIL in resting conditions and upon stimulation with TLR2/TLR3/TLR4 ligands. Unswitched IgD + B cells were co-cultured with RASF/OASF/RADF in the presence/absence of TLR ligands and with/without $\mathrm{BAFF} / \mathrm{APRIL}$ blocking antibodies. Activation-induced cytidine deaminase (AID) mRNA expression, I $\mathrm{C}$ and I -C circular transcripts (CTs; markers of ongoing class-switching to IgG and IgA) and IgM/A/G production were measured to assess functional activation of B cells. RESULTS: TLR3 and to a lesser extent TLR4, but not TLR2 stimulation, induced up to 1000-fold BAFF mRNA and increased soluble BAFF release. APRIL was less significantly regulated by TLR3. Resting and TLR3-stimulated RASF released higher levels of BAFF/APRIL compared with RADF. TLR3 stimulation of RASF but not RADF in co-culture with B cells strongly enhanced AID expression, I-C and I-C CTs and class-switching to IgG/IgA. Blockade of BAFF/APRIL signalling completely inhibited TLR3-induced, RASF-dependent expression of AID, CTs and the secretion of IgG/IgA. CONCLUSIONS: RASF produce high levels of BAFF and APRIL constitutively and in response to TLR3 stimulation. These factors are critical in directly modulating AID expression, class-switch recombination and $\operatorname{IgG} / \operatorname{IgA}$ production in $\operatorname{IgD}+\mathrm{B}$ cells. Overall, this work highlights a novel and fundamental role for the TLR3/B cell survival factor axis in sustaining B cell activation in the rheumatoid arthritis synovium.
\end{abstract}

DOI: https://doi.org/10.1136/ard.2011.150219

Posted at the Zurich Open Repository and Archive, University of Zurich

ZORA URL: https://doi.org/10.5167/uzh-52989

Journal Article

Accepted Version

Originally published at:

Bombardieri, M; Kam, N W; Brentano, F; Choi, K; Filer, A; Kyburz, D; McInnes, I B; Gay, S; Buckley, C; Pitzalis, C (2011). A BAFF/APRIL-dependent TLR3-stimulated pathway enhances the capacity of rheumatoid synovial fibroblasts to induce AID expression and Ig class-switching in B cells. Annals of the Rheumatic Diseases, 70(10):1857-1865.

DOI: https://doi.org/10.1136/ard.2011.150219 


\section{A BAFF/APRIL-dependent TLR3-stimulated pathway enhances the capacity of rheumatoid synovial fibroblasts to induce AID expression and Ig class-switching in B cells}

Michele Bombardieri ${ }^{1}$, Yvonne Ngar Woon $\mathrm{Kam}^{1}{ }^{*}$, Fabia Brentano $^{2}$, Ken $\mathrm{Choi}^{1}$, Andrew Filer $^{3}$, Diego Kyburz ${ }^{2}$, Iain B McInnes ${ }^{4}$, Steffen Gay $^{2}$, Christopher Buckley ${ }^{3}$, Costantino Pitzalis $^{1}$

${ }^{1}$ Centre for Experimental Medicine and Rheumatology, Queen Mary University of London, William Harvey Research Institute, London, UK

${ }^{2}$ Centre for Experimental Rheumatology, University Hospital, Zürich, Switzerland.

${ }^{3}$ Division of Immunity and Infection, College of Medical and Dental Sciences, University of Birmingham, UK

${ }^{4}$ Centre for Rheumatic Diseases, Division of Immunology, Infection and Inflammation, Glasgow Royal Infirmary, University of Glasgow, UK

* The First Authors contributed equally

Corresponding Author:

Dr Michele Bombardieri, MD PhD

Centre for Experimental Medicine and Rheumatology

2nd Floor, John Vane Science Centre

William Harvey Research Institute

Queen Mary University of London

Charterhouse Square

London EC1M 6BQ 
Tel: +44 (0)20 78828193

e.mail: m.bombardieri@qmul.ac.uk

Keywords: Synovial Fibroblasts, B cells, Activation-induced cytidine deaminase, TLR, BAFF

The Corresponding Author has the right to grant on behalf of all authors and does grant on behalf of all authors, an exclusive licence (or non exclusive for government employees) on a worldwide basis to the BMJ Publishing Group Ltd to permit this article (if accepted) to be published in ARD and any other BMJPGL products and sublicences such use and exploit all subsidiary rights, as set out in our licence). 
ABSTRACT

Objectives. To dissect the role of TLR signaling and B cell survival/proliferating factors in the cross-talk between rheumatoid synovial fibroblasts (RASF) and B cells.

Methods. RASF, RA dermal fibroblasts (RADF) and SF from OA patients (OASF), were analysed for the expression of B cell survival/proliferating factors BAFF and APRIL in resting conditions and upon stimulation with TLR2/TLR3/TLR4 ligands. Un-switched IgD+ B cells were co-cultured with RASF/OASF/RADF in the presence/absence of TLR ligands and with/without BAFF/APRIL blocking antibodies. AID mRNA expression, $\mathrm{I} \gamma$-C $\mu$ and I $\alpha$ $\mathrm{C} \mu$ circular transcripts (CTs, markers of ongoing class-switching to $\operatorname{IgG}$ and $\operatorname{IgA}$ ) and $\mathrm{IgM} / \mathrm{A} / \mathrm{G}$ production were measured to assess functional activation of $\mathrm{B}$ cells.

Results. TLR3 and to a lesser extent TLR4, but not TLR2 stimulation induced up to 1000 fold BAFF mRNA and increased soluble BAFF release. APRIL was less significantly regulated by TLR3. Resting and TLR3-stimulated RASF released higher levels of BAFF/APRIL compared to RADF. TLR3 stimulation of RASF but not RADF in co-culture with B cells strongly enhanced AID expression, I $\gamma-\mathrm{C} \mu$ and $\mathrm{I} \alpha-\mathrm{C} \mu \mathrm{CT}$ s and class-switching to IgG/IgA. Blockade of BAFF/APRIL signaling completely inhibited TLR3-induced RASFdependent expression of AID, CTs and the secretion of $\mathrm{IgG} / \mathrm{IgA}$.

Conclusions. RASF produce high levels of BAFF and APRIL constitutively and in response to TLR3 stimulation. These factors are critical in directly modulating AID expression, CSR and $\mathrm{IgG} / \mathrm{Ig} \mathrm{A}$ production in $\mathrm{IgD}+\mathrm{B}$ cells. Overall, we highlight a novel and fundamental role for the TLR3/B cell survival factor axis in sustaining B cell activation in the RA synovium. 


\section{INTRODUCTION}

Rheumatoid arthritis (RA) is a chronic autoimmune/inflammatory disease characterised by immune cell infiltration in the synovial tissue leading to cartilage destruction and bone erosion[1]. One of the hallmarks of RA is a dysregulated activation of autoreactive B cells, leading to the production of autoantibodies such as rheumatoid factor (RF) and anticitrullinated protein antibodies (ACPA), which are important diagnostic and prognostic markers and are implicated in disease pathogenesis[2]. The critical relevance of B cells in RA pathogenesis is demonstrated by the clinical effectiveness of B cell depletion[3,4]. However, the cellular and molecular mechanisms regulating autoreactive B cell activation in RA are poorly understood. We and others have shown that RA synovial tissues with ectopic lymphoid structures (ELS), characterised by perivascular aggregates of $\mathrm{T}$ and $\mathrm{B}$ cells with the differentiation of follicular dendritic cells (FDC) networks[5,6], represent functional niches of autoreactive $\mathrm{B}$ cell activation and differentiation. In particular, ELS promote the expression of activation-induced cytidine deaminase (AID), the enzyme required for somatic hypermutation (SHM) and class-switch recombination (CSR) of the Ig genes, triggering synovial B cells to undergo in situ differentiation towards autoreactive plasma cells $[7,8]$. ELS+ RA synovia engrafted into severe combined immunodeficient (SCID) mice sustain AID expression, B cell survival and proliferation, ongoing CSR and production of ACPA in the absence of re-circulating immune cells[8], suggesting that resident synovial cells are critically involved in regulating humoral autoimmunity in RA. B cell functionality in the SCID/RA model is critically dependent on the B cell survival and proliferating factors BAFF and APRIL[8,9], although the source of these factors in the synovial microenvironment is unclear.

RA fibroblast-like synoviocytes (RASF) share properties of FDC in rescuing B cells from apoptosis and promoting their proliferation and differentiation into antibody-producing 
cells[10-14]. However, the mechanisms regulating the cross-talk between RASF and B cells are unclear. Recent evidence demonstrated that B cells can be directly activated to undergo Ig class-switching in the presence of BAFF and APRIL released at mucosal sites by epithelial cells sensing pathogens via Toll-like receptors (TLR), particularly TLR3[15,16]. RASF can express high levels of BAFF and APRIL[17,18], although the effective capacity of these factors in supporting AID expression and ongoing CSR is unclear. In addition, although accumulating evidence suggests that the activation state of RASF is critically dependent on the expression of TLRs[19-21], the relationship between TLRs and BAFF/APRIL expression in RASF is controversial[19-21].

In the present work we investigated the effect of TLR2/3/4 ligands in modulating BAFF/APRIL expression in RASF and their role in promoting AID expression and CSR in un-switched $\operatorname{IgD}+\mathrm{B}$ cells. 


\section{MATERIALS AND METHODS}

\section{$\underline{\text { Synovial tissue collection }}$}

Synovial tissues were obtained from patients undergoing total knee/hip replacement. A total of 6 RA and 8 OA samples were available at QMUL and used for isolation of synovial fibroblasts. Samples were collected after informed consent (LREC07/Q0605/29 granted by the East London \& The City Research Ethics Committee 3). Matched synovial and skin fibroblasts from 5 RA patients undergoing total knee/hip replacement were obtained from the University of Birmingham (LREC5735), as previously reported[22]. All RA patients (10F/1M, mean age $64.1 \mathrm{yrs}, 82 \% \mathrm{RF}$ positive) fulfilled the American College of Rheumatology criteria for the classification of RA[23] and had long-standing disease requiring joint replacement. All patients had received DMARDs (in monotherapy or combination) and 2 patients received anti-TNF therapy. Patients had discontinued treatment before surgery for an appropriate washout period. Patients with OA had advanced disease and were all diagnosed with primary OA.

\section{$\underline{\text { Generation of fibroblast-like synoviocytes from RA and OA patients }}$}

RASF and OASF were obtained as previously described[24]. After discarding fat and dense fibrous tissues, synovium was minced, mashed under 70- $\mu$ m nylon mesh cell strainer (Becton Dickinson) and digested overnight at $37^{\circ} \mathrm{C}$ with $1.5 \mathrm{mg} / \mathrm{ml}$ Dispase II in DMEM supplemented with $10 \%$ FCS, $50 \mathrm{IU} / \mathrm{mL}$ penicillin-streptomycin, $10 \mathrm{mM}$ HEPES buffer (Gibco/Invitrogen)]. The resulting suspension was passed through cell strainer, centrifuged and the cell pellet re-suspended in culture medium and incubated in T75 tissue-culture flasks. When $90 \%$ confluent SF were passaged $1 / 3$ using $0.25 \%$ trypsin/EDTA (Sigma, UK). 
Synoviocytes were used between passages 4-8 when the culture is devoid of contaminating lymphocyte and macrophages[25].

$\underline{\text { Purification of un-switched } \operatorname{IgD}^{+} \text {human B cells }}$

Human B cells were immunomagnetically selected from tonsils obtained following routine tonsillectomy. Tonsils were washed with chilled $\left(4^{\circ} \mathrm{C}\right)$ MACS buffer [PBS, $0.5 \%$ FCS and 2mM EDTA], minced and gently mashed through a cell strainer. Cells were washed, centrifuged and incubated with $0.5 \mu \mathrm{g} / 10^{6}$ cells of an anti-IgD biotinylated antibody (Southern Biotechnologies) for $30 \mathrm{~min}$ on ice. After washing, cells were incubated with anti-biotin MicroBeads (Miltenyi Biotec) for $15 \mathrm{~min}$ at $4^{\circ} \mathrm{C}$, washed and passed through magnetic separation columns (MS; Miltenyi Biotec). The effluent was collected as flow through (FT) fractions and the bead-bound cells (after-sorted, AS) were collected as enriched, positively selected $\operatorname{IgD}^{+} \mathrm{B}$ cells. All sorting procedures were performed under sterile conditions. The purity of $\operatorname{IgD}+$ unswitched B cell isolation was checked by FACS (Supplementary Figure 1).

\section{TLR stimulation of RASF, OASF and RADF}

Fibroblasts were seeded into 24 -well plates at $4 \times 10^{4}$ cells/well and stimulated at $90 \%$ confluence with optimal concentrations of TLR2-4 ligands [19] (300ng/ml of bacterial

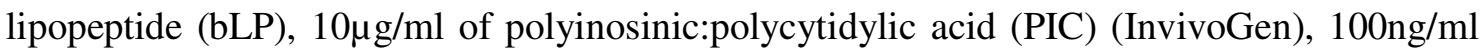
of lipopolysaccharide (LPS) from Escherichia coli J5 (Sigma) or left untreated. Supernatants were harvested after the allocated time period, centrifuged and stored at $-20^{\circ} \mathrm{C}$ until tested. For RNA extraction, adherent fibroblasts were rinsed with PBS and lysed in RLT buffer.

\section{Co-cultures of B cells with RASF, OASF and RADF}

Fibroblasts were seeded into 24-well plates $\left(4 \times 10^{4}\right.$ cells $/ 1 \mathrm{ml} /$ well $)$ and used for co-culture experiments at $90 \%$ confluence. In cell-cell contact experiments, IgD+ B cells $\left(1 \times 10^{6}\right.$ 
cells $/ 300 \mu \mathrm{l} /$ well) were added onto the fibroblast layer with or without $10 \mu \mathrm{g} / \mathrm{ml}$ PIC. In transwell experiments, microporous PET cell inserts (1- $\mu \mathrm{m}$ pore, Beckon Dickinson) were employed. B cells were grown in the upper well and PIC treated or untreated fibroblasts were grown on the base of the well. Control co-cultures received no fibroblasts in order to investigate baseline expression in isolated $\mathrm{IgD}+\mathrm{B}$ cells. Co-cultures were kept for $24 \mathrm{~h}, 72 \mathrm{~h}$ or 8 days, depending on the experimental conditions after which cell-free supernatants and B cells were collected. For gene expression profiling, pelleted B cells were lysed in RLT buffer (Qiagen).

Blockade of BAFF/APRIL signaling on B cells with anti-BCMA and anti-BAFFR $\underline{\text { neutralizing antibodies }}$

BAFF/APRIL dependent signalling in B cells was blocked using neutralizing polyclonal goat anti-human BAFFR and anti-human BCMA antibodies (R\&D Systems) at 10 $\mu \mathrm{g} / \mathrm{ml}$ alone or in combination, $30 \mathrm{~min}$ before co-culture and throughout the experiment. After $72 \mathrm{~h}$ or 8 days of co-culture, supernatants and cells were harvested as described above.

\section{$\underline{\text { Gene expression analysis by quantitative real-time PCR (QT-PCR) }}$}

Total RNA was isolated using the RNeasy Mini kit (Qiagen). RNA (500ng) was reverse transcribed to cDNA using the Thermoscript RT-PCR System (Invitrogen). For gene expression profiling QT-PCR was performed on cDNAs using specific TaqMan primers and probes [8] (Applied Biosystems, Supplementary Table 1). Taqman QT-PCR was run in triplicate with 10ng/well at 40 cycles of amplifications in an ABI $7900 \mathrm{HT}$ Instrument. Human $18 \mathrm{~S}$ and human B-actin were used as endogenous controls. Relative quantification was measured using the Comparative $\mathrm{C}_{\mathrm{T}}$ (Threshold Cycle) Method. 
Detection of circular transcripts (CTs) by polymerase-chain reaction (PCR)

Detection of $\mathrm{I} \gamma_{1 / 2}-\mathrm{C} \mu$ and $\mathrm{I} \alpha-\mathrm{C} \mu \mathrm{CTs}$ were performed as previously described with minor modifications [8] using $0.5 \mathrm{U}$ of Taq polymerase (MP biomedicals)/20 $\mu 1$ reaction volume. Primers sequences and PCR conditions are summarized in Supplementary Table 2. After final extension at $72^{\circ} \mathrm{C}$ for $5 \mathrm{~min}$, PCR products were run on $1 \%$ agarose gel $/ 0.5 \mu \mathrm{g} / \mathrm{ml}$ ethidium bromide in TAE buffer and visualised under UV light.

\section{$\underline{\text { ELISA }}$}

BAFF and APRIL protein concentration in cell-free supernatants was measured using the Quantikine Human BAFF/BLyS/TNFSF13B (R\&D Systems) and Human APRIL (eBioscience) ELISA kits respectively, following the manufacturers' instructions.

Total IgA, IgG and IgM were detected using the Human $\operatorname{Ig} \mathrm{A} / \operatorname{IgG} / \operatorname{IgM}$ ELISA quantitation set (Bethyl laboratories) according to manufacturer's instruction.

\section{Detection of BAFF and APRIL expression in fibroblasts using immunocytochemistry}

Fibroblasts were seeded at $5 \times 10^{4}$ cells/200 $\mu 1$ onto cover slides and grown to $90 \%$ confluence. Cells were then stimulated with $10 \mu \mathrm{g} / \mathrm{ml}$ PIC or left untreated. After $24 \mathrm{~h}$, cells were washed in PBS and fixed using ice-cold 1:1 acetone/methanol. After washing in TBS and blocking with avidin-biotin blocking kit (Vector laboratory) and DAKO serum-free protein block, rat anti-human BAFF (Buffy-2, Enzo Life Sciences), mouse anti-human APRIL (Aprily-2, Enzo Life Sciences) or isotype controls at $10 \mu \mathrm{g} / \mathrm{ml}$ were applied for $1 \mathrm{~h}$ at RT. After washing, biotinylated donkey anti-rat (Serotec) or rabbit anti-mouse (Dako) antibodies were added for $1 \mathrm{~h}$ at RT. After washing, slides were incubated for 30min with Streptavidin-ALEXA555 or Streptavidin-FITC (1:300 dilution) and counterstained with DAPI. Slides were examined under Olympus BX60 microscope. 


\section{$\underline{\text { Statistical Analysis }}$}

Differences in quantitative variables were analysed by the Mann Whitney $U$ test when comparing 2 groups and by Kruskal-Wallis with Dunn's post test when comparing multiple groups. Wilcoxon's signed-rank test was used to analyse repeated measurements at different time-points. All analyses were performed using GraphPad Prism version 5.01, GraphPad Software, USA. A p value <0.05 was considered statistically significant. 


\section{RESULTS}

TLR3-stimulated induction of BAFF and APRIL mRNA in RASF, OASF and RADF

We first examined whether TLR stimulation of RASF and OASF (to dissect disease specificity) and paired RADF (to investigate site specificity) induced BAFF and APRIL mRNA. At baseline, RASF and OASF displayed increased expression of BAFF mRNA, between 4 and 8-fold higher than RADF (data not shown), as previously reported[17]. BAFF mRNA expression in RASF and OASF was markedly induced by PIC (Figure 1a, e), whilst there was a more modest induction with LPS and no upregulation with bLP. PIC triggered a rapid induction of BAFF transcripts at $4 \mathrm{~h}$, which peaked at $8 \mathrm{~h}$ and gradually decreased afterwards. A comparable increase in BAFF mRNA was induced by PIC in RADF (Figure 1c). Interestingly, RADF produced significantly higher levels of BAFF mRNA in response to TLR4 stimulation as compared to RASF or OASF, and similar to that obtained with PIC.

In comparison with BAFF, APRIL was significantly but more modestly modulated by TLR3 stimulation in RASF and OASF ( $\sim$-fold increase) (Figure $1 b, f)$ with no apparent modulation in RADF (Figure 1d), and was characterised by a later peak of induction at $24 \mathrm{~h}$. We finally investigated whether there was a differential expression of TLR3 mRNA in RASF compared to RADF and we found no difference among these cells in basal TLR3 expression (Supplementary Figure 3).

\section{$\underline{\text { RASF but not RADF release high levels of BAFF in response to TLR3 stimulation }}$}

We next examined whether TLR3-induced BAFF mRNA was mirrored by increased protein expression. As shown in Figure 2a, high amounts of soluble BAFF were released in the supernatant of RASF in a time-dependent manner and were significantly increased upon PIC stimulation, (Figure 2a). Similar levels were observed in the supernatant of OASF (data not 
shown). Despite strong up-regulation of BAFF mRNA, RADF produced only modest amount of soluble BAFF in resting conditions and in response to PIC.

We next investigated the intracellular localization of BAFF upon TLR3 stimulation by immunofluorescence. As shown in Figure $2 \mathrm{~b}$, weak and granular perinuclear BAFF immunoreactivity was detected in untreated permeabilized RASF. Upon incubation with PIC a strong increase in cytoplasmic BAFF (Figure 2b) as well as membrane-bound BAFF (Supplementary Figure 4) was observed. Similar data were observed in OASF (data not shown). In contrast, following exposure to PIC, BAFF expression remained barely detectable in RADF. Overall, these data indicate that increased production of BAFF, at basal level or upon TLR3 stimulation, is characteristic of SF but not DF.

\section{$\underline{\text { RASF but not RADF constitutively produce large amounts of APRIL }}$}

We next investigated the ability of RASF and RADF to release APRIL in resting and stimulated conditions. Unlike RADF, RASF produced abundant basal levels of soluble APRIL, reaching $\sim 900 \mathrm{pg} / \mathrm{ml}$ on day 3 (Figure 3a) which was only marginally increased upon TLR3 stimulation (Figure 3a). However, APRIL was released at a higher rate within the first 24h in PIC-stimulated RASF. Again, similar results were observed in OASF (data not shown). In contrast to RASF, APRIL levels in RADF were extremely low in basal conditions and remained low following TLR3 activation. Immunofluorescence staining for APRIL demonstrated that while resting RASF expressed an intense perinuclear and cytoplasmic immunoreactivity (Figure 3b), likely corresponding to vesicle-sequestered or Golgiassociated APRIL [26], RADF were characterised by a significantly weaker staining. Overall, our findings indicated that RASF, but not RADF, retain the capacity to release constitutively high levels of APRIL which are modestly increased upon TLR3 stimulation. 
TLR3 stimulation of RASF, but not RADF, enhances class-switched immunoglobulin $\underline{\text { secretion from IgD+ human B lymphocytes }}$

We next examined whether TLR3 stimulation enhanced the capacity of RASF to promote Ig production in B lymphocytes[12] and whether this ability was dependent on direct cell-cell interactions and/or soluble factors.

Tonsil IgD+ un-switched B cells at 97\% purity (Supplementary Figure 1) were co-cultured either in cell-cell contact or in a transwell system with RASF, OASF or RADF. As shown in Figure 4, $\operatorname{IgD}^{+}$B cells alone produced low amounts of $\operatorname{IgG}$ and $\operatorname{IgA}$ (Figure 4a). Conversely, co-culture of B cells with RASF induced a 2-3 fold increase in IgG production already at $72 \mathrm{~h}$ which was significantly increased upon TLR3 stimulation (Figure 4a). Similar, albeit more modest induction of IgA was also evident. Paired RADF did not induce IgG or IgA production either in resting or stimulated conditions (Figure 4b), while OASF displayed a similar behaviour compared to RASF (data not shown). Interestingly, CSR to $\operatorname{IgG} / \operatorname{IgA}$ still occurred (albeit with slightly lower efficiency) when B cells were cultured in transwells (Figure 4a), indicating that soluble factors released by RASF are sufficient to promote CSR. Treatment of RASF with PIC minimally modulated IgM production, suggesting that RASF induced functional B cell activation and not simply increased survival/proliferation, as indicated by the increased IgG/IgM ratio after 8 days compared to $24 \mathrm{~h}$ and $72 \mathrm{~h}$ (Figure $5 \mathrm{~d}$ ).

TLR3-stimulated RASF induce up-regulation of AID and circular transcripts in B cells A formal demonstration of the capacity of RASF to induce class-switching require evidence of AID expression and the appearance of circular transcripts (CTs). RASF strongly induced AID mRNA after 24 and $72 \mathrm{~h}$ of coculture in cell-cell and transwell systems, a capacity further enhanced upon TLR3 stimulation (Figure 5a, b). $\operatorname{IgD}^{+} \mathrm{B}$ cells alone (Figure 5a, b) or cultured with RADF (data not shown) displayed no or barely detectable levels of AID 
mRNA. The specificity of the AID and endogenous control PCR products were confirmed by agarose gel electrophoresis (Supplementary Figure 2c). In order to confirm that AID was functional, we determined the presence of $\gamma_{1 / 2}-\mathrm{C} \mu \mathrm{CT}$, specific by-products of ongoing CSR from IgM to IgG1/2 in B cells, which disappear $48 \mathrm{~h}$ after CSR[27]. While no $\mathrm{I} \gamma_{1 / 2}-\mathrm{C} \mu \mathrm{CTs}$ were detected in $\operatorname{IgD}+\mathrm{B}$ cells alone at $72 \mathrm{~h}$, co-culture with unstimulated RASF was sufficient to induce CTs expression which was clearly enhanced upon TLR3 stimulation (Figure 5c, top gel, lane 4). RASF-dependent induction of $\mathrm{I} \gamma_{1 / 2}-\mathrm{C} \mu \mathrm{CTs}$ and IgG production was similar to that observed using recombinant BAFF and IL4 (Supplementary Figure 2).

Overall, these data provide conclusive evidence that RASF directly induce the expression of AID and the molecular machinery required for ongoing CSR in un-switched B cells and that TLR3 stimulation enhances this effect.

Dual blockade of BAFFR and BCMA abrogates TLR3-induced AID expression and classswitching

We finally investigated whether the activating capacity of RASF in B cells in response to TLR3 was dependent on BAFF and/or APRIL. By using neutralising antibodies against BAFFR (specific for BAFF) and BCMA (binding both BAFF and APRIL) we showed that in the transwell system individual receptor blocking displayed significant but partial inhibition of TLR3-dependent IgG and IgA production, while the combination of anti-BAFFR/antiBCMA completely suppressed TLR3-induced IgG and IgA production (Figure 6a). In addition, blockade of BAFFR/BCMA down-regulated AID expression and the production of I $\gamma_{1 / 2}-\mathrm{C} \mu \mathrm{CTs}$ (Figure 6 b-c respectively). Experiments in cell-cell contact displayed similar results, although the inhibitory effect was slightly smaller (data not shown). Overall, these findings suggest that CSR induced in IgD+ B cells by RASF via TLR3 stimulation is mostly 
dependent on the production of BAFF and APRIL and can be blocked by receptor interference. 


\section{DISCUSSION}

In situ autoreactive B cell activation and autoantibody production within the RA synovium is a typical feature of RA patients in which synovitis is characterised by the formation of ectopic lymphoid structures (ELS). Within RA lymphoid aggregates, B lymphocytes undergo antigen-driven affinity maturation, clonal expansion, Ig isotype switching and plasma cell differentiation[7,28,29]. We recently showed that these processes i) require the expression of AID, the enzyme responsible for the initiation of SHM and CSR of the Ig genes, ii) are selfsustained for several weeks in the SCID/RA chimeric model in the absence of recirculating immune cells and iii) develop in the context of prolonged expression of the B cells survival and proliferating factors BAFF and APRIL[8]. The functionality of synovial B cell follicles is dependent on the interaction with networks of stromal cells which express CD21[5,6] and closely resemble FDC in secondary lymphoid organs[30]. Accordingly, the diffuse form of synovitis, characterised by the absence of B cell aggregates, lacks AID expression and ongoing Ig class-switching[8]. Despite strong evidence that ELS support the production of autoantibodies within the RA synovium, we and other demonstrated that circulating autoantibodies in RA do not associate with the presence of synovial ELS [8,31,32], suggesting that secondary lymphoid organs are also important sites for autoreactive B cell activation.

Although it is currently unclear whether synovial FDC differentiate from resident stromal cells[33], fibroblast-like synoviocytes isolated from the RA synovium (RASF) are able to rescue $\mathrm{B}$ cells from apoptosis and promote their differentiation into antibody-producing cells[10-13,34,35]. However, the critical factors regulating these interactions have not been fully investigated. Cell-dependent mechanisms involving adhesion molecules (i.e. VCAM-1) and chemokines such as CXCL12 only partially account for the observed B cell activation[10], suggesting a critical contribution of additional factors. In addition, because 
previous work did not differentiate between naïve, switched and unswitched memory B cells in co-culture experiments, the actual capacity of RASF to induce the molecular machinery required for Ig class-switching in naïve B cells was questionable.

In this work, we provide novel observations which contribute to our understanding of the mechanisms regulating the cross-talk between RASF and B cells within the RA synovium. First, we demonstrated that RASF constitutively produce large amount of BAFF and APRIL, expanding recent evidence of over-expression of these factors at mRNA level in RASF[17,18,36]. Furthermore, we showed that TLR3 stimulation induced strong and rapid up-regulation of BAFF at both mRNA and protein level while APRIL was more modestly modulated and displayed a delayed kinetic of expression, peaking 24-48h post-stimulation. Interestingly, soluble BAFF levels in resting and TLR3-activated RASF were comparable to those released by dendritic cells and airway epithelial cells, which express biologically functional amounts of BAFF upon TLR3 triggering[37-39]. Similarly, in this work we provided the first demonstration that RASF constitutively release high levels of soluble APRIL. Although, TLR3 activation did not induce a large increase in the amount of APRIL produced by RASF, APRIL was released at higher rate within the first $24 \mathrm{~h}$, possibly reflecting a role for TLR3 in the early mobilization of intracellular APRIL. Furthermore, APRIL was not homogeneously expressed among RASF, demonstrating some heterogeneity within the RASF population.

Despite RASF over-express TLR4[19], LPS treatment induced only limited levels of BAFF, similar to airway fibroblasts[40]. These results are in keeping with previous evidence that TLR4 can even down-regulate BAFF mRNA expression in RASF in the presence of IFN $\gamma[21]$ and confirm that BAFF transcription is under the control of TRIF (Toll/IL-1R domain-containing protein inducing IFN- $\beta$ ) rather than MyD88-dependent pathways. Importantly, the capacity of synoviocytes to respond to TLR3 by releasing high levels of 
BAFF/APRIL was not restricted to RASF as OASF displayed similar expression in resting and stimulated conditions. Conversely, RADF produced negligible amounts of BAFF/APRIL and failed to significantly increase protein expression despite similar mRNA responses to TLR3. Overall, this suggests that the over-expression of BAFF and APRIL in SF is dependent on epigenetic abnormalities related to the anatomical localization rather than disease specificity[22]. In addition, the importance of TLR3 is relevant to RA pathogenesis as RASF can be activated in the synovial microenvironment by RNA released by necrotic cells [24]. Importantly, because TLR3 signals via the adaptor molecule TRIF in a MyD88independent pathway, its activation also leads to type I IFN and IFN-responsive genes expression. In this regard, increase in IFN $\beta$, which is known to be upregulated in the RA synovium [41], might contribute to the pathogenesis of RA by exacerbating Th17, as recently suggested [42].

RASF were extremely efficient compared to RADF in inducing the prompt and sustained production of class-switched antibodies, primarily $\operatorname{IgG}$ and to a lesser extent $\operatorname{Ig} \mathrm{A}$ from unswitched IgD+ B cells. Stimulation of RASF (and OASF), but not RADF, with TLR3 ligands strongly enhanced the production of $\operatorname{IgG}$ and $\operatorname{IgA}$ in co-cultured B cells, an effect directly dependent on RASF as IgD+ B cells alone did not respond to PIC.

The process of isotype class-switching requires AID and occurs via excision of switch circles following the introduction of double strand DNA breaks in the Ig switch regions[43]. Accordingly, we showed that RASF, but not RADF, induced a rapid and sustained de novo expression of AID in co-cultured IgD+ B cells, an effect significantly potentiated by TLR3 simulation. Subsequently, we demonstrated that AID expression induced $\mathrm{I} \gamma$-C $\mu$ CTs, which are a sensitive marker of class-switching, being transiently produced for up to $48 \mathrm{~h}$ after CSR[27]. Thus, increased detection of $\mathrm{I} \gamma$-C $\mu \mathrm{CTs}$ after over $72 \mathrm{~h}$ of co-culture demonstrated that class-switching from $\operatorname{IgM}$ to $\operatorname{IgG}$ was actively ongoing and that TLR3 stimulation of 
RASF potentiated CSR in $\operatorname{IgD}+\mathrm{B}$ cells. Accordingly, the $\mathrm{IgG} / \mathrm{IgM}$ ratio was over 10 -fold higher 8 days after co-culture in TLR3-stimulated conditions as compared to 24h. Of relevance, TLR3-induced RASF-dependent B cells activation was maintained when B cells and RASF were separated via a cell insert, suggesting that soluble factors are sufficient to induce B cell activation. Thus is of particular importance as BAFF can elicit its biological functions both in a membrane-bound and a soluble form[44].

BAFF and APRIL are critically involved in promoting functional B cell activation, AID expression and Ig class switching[16,45], thus we finally questioned whether RASF-derived BAFF and APRIL were required to mediate these processes. We showed that TLR3dependent IgG and IgA production was completely inhibited after interfering with signaling through both BAFFR and BCMA on the B cell surface. Of relevance, only minimal effects on IgM production was observed, suggesting that reduced B cell activation rather than survival was responsible for the down-regulation of $\operatorname{IgG/IgA}$. Accordingly, BAFFR/BCMA blockade also down-regulated the expression of AID and the production of I $\gamma$-C $\mu$ CTs. These in vitro data support in vivo observations in the RA/SCID model, in which functional niches of autoreactive B cells required BAFF and APRIL for Ig production[8], AID expression, ongoing class-switching and production of ACPA antibodies[8].

In summary, in this study we report that i) RASF, but not RADF, constitutively release high levels of BAFF and APRIL; ii) BAFF, and to a lesser extent APRIL are induced by TLR3 ligands, iii) TLR3 stimulation enhances the capacity of RASF to promote AID expression, isotype class-switching and $\mathrm{IgG} / \mathrm{IgA}$ production in unswitched $\mathrm{IgD}+\mathrm{B}$ cells; iv) these effects can be efficiently blocked by inhibition of BAFF/APRIL signalling through their receptors on the B cell membrane. Overall, this work contributes to elucidate the mechanisms regulating the cross-talk between stromal and B cells in the synovial microenvironment and highlights the importance of the BAFF/APRIL system in sustaining the functionality of synovial niches 
of autoreactive B cells, with potential implications for biological treatments targeting B cellrelated pathways in RA.

\section{ACKNOWLEDGMENTS}

We would like to thank Dr Taher Taher and Dr Mohey Eldin El Shikh at QMUL for their help with FACS analysis and confocal microscopy respectively. This work was funded by the Arthritis Research UK project grant 18399 to MB, IM and CP and by FP 7 Masterswitch and IAR Epalinges $\mathrm{CH}$ to $\mathrm{FB}, \mathrm{DK}$ and $\mathrm{SG}$. 


\section{REFERENCES}

1. Magalhaes R, Stiehl P, Morawietz L et al. Morphological and molecular pathology of the $\mathrm{B}$ cell response in synovitis of rheumatoid arthritis. Virchows Arch. 2002;441:415-7.

2. van Venrooij WJ, van Beers JJ, Pruijn GJ. Anti-CCP Antibody, a Marker for the Early Detection of Rheumatoid Arthritis. Ann N Y Acad Sci. 2008;1143:268-85.

3. Vallerskog T, Heimburger M, Gunnarsson I et al. Differential effects on BAFF and APRIL levels in rituximab-treated patients with systemic lupus erythematosus and rheumatoid arthritis. Arthritis Res Ther. 2006; 8:R167.

4. Edwards JC, Szczepanski L, Szechinski J et al. Efficacy of B-cell-targeted therapy with rituximab in patients with rheumatoid arthritis. N Engl J Med. 2004;350:257281.

5. Takemura S, Braun A, Crowson $\mathrm{C}$, et al. Lymphoid neogenesis in rheumatoid synovitis. J Immunol. 2001;167:1072-80.

6. Manzo A, Paoletti S, Carulli M, et al. Systematic microanatomical analysis of CXCL13 and CCL21 in situ production and progressive lymphoid organization in rheumatoid synovitis. Eur J Immunol. 2005;35:1347-59.

7. Kim HJ, Krenn V, Steinhauser G, et al. Plasma cell development in synovial germinal centers in patients with rheumatoid and reactive arthritis. J Immunol. 1999;162:305362.

8. Humby F, Bombardieri M, Manzo A, et al. Ectopic lymphoid structures support ongoing production of class-switched autoantibodies in rheumatoid synovium. PLoS Med. 2009;6(1):e1.

9. Seyler TM, Park YW, Takemura S, B et al. BLyS and APRIL in rheumatoid arthritis. J Clin Invest. 2005;115:3083-92.

10. Burger JA, Zvaifler NJ, Tsukada N, et al. Fibroblast-like synoviocytes support B-cell pseudoemperipolesis via a stromal cell-derived factor-1- and CD106 (VCAM-1)dependent mechanism. J Clin Invest. 2001;107:305-15.

11. Edwards JC, Leigh RD, Cambridge G. Expression of molecules involved in B lymphocyte survival and differentiation by synovial fibroblasts. Clin Exp Immunol. 1997;108:407-14.

12. Dechanet J, Merville P, Durand I, et al. The ability of synoviocytes to support terminal differentiation of activated B cells may explain plasma cell accumulation in rheumatoid synovium. J Clin Invest. 1995;95:456-63. 
13. Shimaoka Y, Attrep JF, Hirano T, et al. Nurse-like cells from bone marrow and synovium of patients with rheumatoid arthritis promote survival and enhance function of human B cells. J Clin Invest. 1998;102:606-18.

14. Pap T, Muller-Ladner U, Gay RE, et al. Fibroblast biology. Role of synovial fibroblasts in the pathogenesis of rheumatoid arthritis. Arthritis Res. 2000;2:361-7.

15. $\mathrm{Xu} \mathrm{W}, \mathrm{He} \mathrm{B}$, Chiu A, et al. Epithelial cells trigger frontline immunoglobulin class switching through a pathway regulated by the inhibitor SLPI. Nat Immunol. 2007;8:294-303.

16. $\mathrm{He} \mathrm{B}, \mathrm{Xu} \mathrm{W}$, Santini $\mathrm{PA}$, et al. Intestinal bacteria trigger $\mathrm{T}$ cell-independent immunoglobulin $\mathrm{A}(2)$ class switching by inducing epithelial-cell secretion of the cytokine APRIL. Immunity. 2007;26:812-26.

17. Ohata J, Zvaifler NJ, Nishio M, et al. Fibroblast-like synoviocytes of mesenchymal origin express functional B cell-activating factor of the TNF family in response to proinflammatory cytokines. J Immunol. 2005;174:864-70.

18. Nagatani $\mathrm{K}$, Itoh $\mathrm{K}$, Nakajima $\mathrm{K}$, et al. Rheumatoid arthritis fibroblast-like synoviocytes express BCMA and are stimulated by APRIL. Arthritis Rheum. 2007;56:3554-63.

19. Ospelt C, Brentano F, Rengel Y, et al. Overexpression of toll-like receptors 3 and 4 in synovial tissue from patients with early rheumatoid arthritis: toll-like receptor expression in early and longstanding arthritis. Arthritis Rheum. 2008;58:3684-92.

20. Neumann E, Lefevre S, Zimmermann B, et al. Rheumatoid arthritis progression mediated by activated synovial fibroblasts. Trends Mol Med. 2010;16:458-48.

21. Alsaleh G, Messer L, Semaan N, et al. BAFF synthesis by rheumatoid synoviocytes is positively controlled by alpha5beta1 integrin stimulation and is negatively regulated by tumor necrosis factor alpha and Toll-like receptor ligands. Arthritis Rheum. 2007;56:3202-14.

22. Filer A, Parsonage G, Smith E, et al. Differential survival of leukocyte subsets mediated by synovial, bone marrow, and skin fibroblasts: site-specific versus activation-dependent survival of $\mathrm{T}$ cells and neutrophils. Arthritis Rheum. 2006;54:2096-108.

23. Arnett FC, Edworthy SM, Bloch DA, et al. The American Rheumatism Association 1987 revised criteria for the classification of rheumatoid arthritis. Arthritis Rheum. 1988;31:315-24.

24. Brentano F, Schorr O, Gay RE, et al. RNA released from necrotic synovial fluid cells activates rheumatoid arthritis synovial fibroblasts via Toll-like receptor 3. Arthritis Rheum. 2005;52:2656-65. 
25. Hirth A, Skapenko A, Kinne RW, et al. Cytokine mRNA and protein expression in primary-culture and repeated-passage synovial fibroblasts from patients with rheumatoid arthritis. Arthritis Res. 2002;4:117-25.

26. Lopez-Fraga M, Fernandez R, Albar JP, et al. Biologically active APRIL is secreted following intracellular processing in the Golgi apparatus by furin convertase. EMBO Rep. 2001;2(10):945-51.

27. Kinoshita K, Harigai M, Fagarasan S, et al. A hallmark of active class switch recombination: transcripts directed by I promoters on looped-out circular DNAs. Proc Natl Acad Sci U S A. 2001;98:12620-3.

28. Gause A, Gundlach K, Zdichavsky M, et al. The B lymphocyte in rheumatoid arthritis: analysis of rearranged V kappa genes from B cells infiltrating the synovial membrane. Eur J Immunol. 1995;25:2775-82.

29. Scheel T, Gursche A, Zacher J, et al. V-region gene analysis of locally defined synovial B and plasma cells reveals selected B cell expansion and accumulation of plasma cell clones in rheumatoid arthritis. Arthritis Rheum. 2011; 63:63-72.

30. Allen CD, Cyster JG. Follicular dendritic cell networks of primary follicles and germinal centers: phenotype and function. Semin Immunol. 2008;20:14-25.

31. Thurlings RM, Wijbrandts CA, Mebius RE, et al. Synovial lymphoid neogenesis does not define a specific clinical rheumatoid arthritis phenotype. Arthritis Rheum. 2008; 58:1582-9.

32. van de Sande MG, Thurlings RM, Boumans MJ, et al. Presence of lymphocyte aggregates in the synovium of patients with early arthritis in relationship to diagnosis and outcome: is it a constant feature over time? Ann Rheum Dis. 2010; 70:700-3.

33. Lindhout E, van Eijk M, van Pel M, et al. Fibroblast-like synoviocytes from rheumatoid arthritis patients have intrinsic properties of follicular dendritic cells. J Immunol. 1999;162:5949-56.

34. Reparon-Schuijt CC, van Esch WJ, van Kooten C, et al. Regulation of synovial B cell survival in rheumatoid arthritis by vascular cell adhesion molecule 1 (CD106) expressed on fibroblast-like synoviocytes. Arthritis Rheum. 2000;43:1115-21.

35. Hayashida K, Shimaoka Y, Ochi T, et al. Rheumatoid arthritis synovial stromal cells inhibit apoptosis and up-regulate Bcl-xL expression by $\mathrm{B}$ cells in a CD49/CD29CD106-dependent mechanism. J Immunol. 2000;164:1110-6.

36. Rochas C, Hillion S, Saraux A, et al. Transmembrane BAFF from rheumatoid synoviocytes requires interleukin-6 to induce the expression of recombinationactivating gene in B lymphocytes. Arthritis Rheum. 2009; 60:1261-71. 
37. Nardelli B, Belvedere O, Roschke V, et al. Synthesis and release of B-lymphocyte stimulator from myeloid cells. Blood. 2001;97:198-204.

38. Kato A, Truong-Tran AQ, Scott AL, et al. Airway epithelial cells produce B cellactivating factor of TNF family by an IFN-beta-dependent mechanism. J Immunol. 2006;177:7164-72.

39. Litinskiy MB, Nardelli B, Hilbert DM, et al. DCs induce CD40-independent immunoglobulin class switching through BLyS and APRIL. Nat Immunol. 2002;3:822-9.

40. Yamada T, Lizhong S, Takahashi N, et al. Poly(I:C) induces BLyS-expression of airway fibroblasts through phosphatidylinositol 3-kinase. Cytokine. 2010;50:163-9.

41. Pilling D, Akbar AN, Girdlestone J, et al. Interferon-beta mediates stromal cell rescue of T cells from apoptosis. Eur J Immunol. 1999; 29:1041-50.

42. Axtell RC, Raman C, Steinman L. Interferon-beta exacerbates Th17-mediated inflammatory disease. Trends Immunol. 2011;Apr 29 [Epub ahead of print].

43. Honjo T, Kinoshita K, Muramatsu M. Molecular mechanism of class switch recombination: linkage with somatic hypermutation. Annu Rev Immunol. 2002;20:165-96.

44. Schneider P, MacKay F, Steiner V, et al. BAFF, a novel ligand of the tumor necrosis factor family, stimulates B cell growth. J Exp Med. 1999;189:1747-56.

45. Castigli E, Wilson SA, Scott S, et al. TACI and BAFF-R mediate isotype switching in B cells. J Exp Med. 2005;201:35-9. 


\section{Figure Legends}

Figure 1. Induction of BAFF and APRIL mRNA in RASF, RADF and OASF in response to TLR ligands.

Quantitative Taqman real-time PCR analysis of BAFF and APRIL mRNA expression in response to TLR ligands. $\operatorname{RASF}(\mathbf{a}, \mathbf{b}), \operatorname{RADF}(\mathbf{c}, \mathbf{d})$ and $\operatorname{OASF}(\mathbf{e}, \mathbf{f})$ were stimulated with 10 $\mu \mathrm{g} / \mathrm{ml}$ PIC (TLR3), 100ng/ml LPS (TLR4), 300ng/ml bLP (TLR2) or medium alone for 4, $8,24,48,72 \mathrm{~h}$. Results are expressed as mean \pm SEM of the mRNA fold increase calculated at each time point by setting the baseline unstimulated expression as 1 . Data were normalized using mammalian $18 \mathrm{~S}$ as an endogenous control gene (18S). $* \mathrm{p}<0.05, * * \mathrm{p}<0.01$ compared to unstimulated

Figure 2. TLR3 stimulation induces intracellular and soluble BAFF in RASF but not RADF.

(a) Column-bar graph showing the amount of soluble BAFF released in the cell culture supernatant from RASF and RADF conditioned for $24,48,72 \mathrm{~h}$ in the presence or absence of $10 \mu \mathrm{g} / \mathrm{ml}$ PIC. RASF produce significantly higher amount of BAFF both in resting and stimulated conditions as compared to RADF. Results are represented as mean \pm SEM of minimum 3 experiments.

(b) Representative microphotographs of BAFF immunostaining on RASF and RADF before and after stimulation with PIC. RASF/RADF were grown on coverslips and processed for immunofluorescence as described in the Methods. Nuclei are counterstained in blue (DAPI) and BAFF in red (Alexa-555). Primary RASF/RADF were left untreated (medium only) or stimulated for $24 \mathrm{~h}$ with PIC $(10 \mu \mathrm{g} / \mathrm{ml})$. Appropriate isotype control was used to confirm 
specificity. Images were captured at $20 \mathrm{X}$ magnification and with the same exposure time for both conditions.

Figure 3. Intracellular and soluble APRIL expression in RASF vs RADF.

(a) Column-bar graph showing the amount of soluble APRIL released in the cell culture supernatant from RASF and RADF conditioned for $24,48,72 \mathrm{~h}$ in the presence or absence of $10 \mu \mathrm{g} / \mathrm{ml}$ PIC. Data are presented as mean \pm SEM of at least 3 experiments.

(b) Representative microphotographs of APRIL immunostaining on RASF and RADF in resting conditions. RASF/RADF were grown on coverslips and processed for immunofluorescence as described in the Methods. Nuclei are counterstained in blue (DAPI) and APRIL is stained in green (Alexa-488). Appropriate isotype control was used to confirm specificity. Images were captured at $20 \mathrm{X}$ magnification and with the same exposure time for both conditions.

Figure 4. TLR3 stimulation of RASF but not RADF enhances their capacity to promote the production of class switched antibodies in IgD+ B cells.

Purified tonsillar $\operatorname{IgD}^{+}$B cells were cultured with fibroblasts for $72 \mathrm{~h}$. B cells were cultured alone (open bars) or in co-culture fibroblasts in direct physical contact (grey bars) or a transwell system (black bar). All cultures were incubated with (+) or without (-) $10 \mu \mathrm{g} / \mathrm{ml}$ PIC. IgG, IgA and IgM levels in supernatants were measured by ELISA. Results are

presented as mean \pm SEM of at least 3 experiments. $* \mathrm{p}<0.05, * * \mathrm{p}<0.01$ compared to unstimulated 
Figure 5. TLR3 stimulation of RASF enhances AID expression and ongoing class switching in co-cultured $\operatorname{IgD}^{+}$tonsillar B cells.

(a-b) Purified tonsillar IgD+ B cells were cultured with RASF for $24 \mathrm{~h}$ (a) and $72 \mathrm{~h} \mathrm{(b)} \mathrm{with}$ $10 \mu \mathrm{g} / \mathrm{ml}$ PIC or left untreated in the presence (transwell) of absence (cell-cell contact) of a cell insert. AID mRNA expression was determined by QT-PCR normalized to an endogenous control gene (18S) and the fold difference relative to baseline expression was calculated by setting AID expression in B cell alone as $1 .{ }^{*} \mathrm{p}<0.05, * * \mathrm{p}<0.01$ compared to unstimulated

(c) Representative $1 \%$ agarose gel (inverted colour) of $\mathrm{I} \gamma-\mathrm{C} \mu$ circular transcripts (CT, amplicon $502 \mathrm{bp}$, upper gel), specific by-products of ongoing class-switching from IgM to IgG. The lower gel represents the endogenous control, mammalian 18S (amplicon 187bp). CTs were amplified from B cell cDNA using specific primers (Methods). B cells were cultured alone or in transwell with RASF for $72 \mathrm{~h}$ in the presence or absence of $10 \mu \mathrm{g} / \mathrm{ml}$ PIC. Tonsil was used as a positive control.

(d) Progressive production of IgG over IgM in B cells co-cultured in transwell with TLR3stimulated RASF after $24 \mathrm{~h}, 72 \mathrm{~h}$ and 8 days, demonstrating. Data are presented as mean IgG to $\operatorname{IgM}$ ratio $\pm \mathrm{SEM}$ of at least 3 experiments per time point. $* \mathrm{p}<0.05, * * \mathrm{p}<0.01$ compared to unstimulated

Figure 6. Blockade of BAFFR and/or BCMA reverses TLR-3 induced RASF-dependent IgD+ $\mathrm{B}$ cell activation.

(a) $\mathrm{IgG}, \operatorname{Ig} \mathrm{A}$ and $\operatorname{IgM}$ production by tonsillar IgD+ B cells cultured in transwell with RASF for $72 \mathrm{~h}$ in the presence of $10 \mu \mathrm{g} / \mathrm{ml}$ of either anti-human BCMA or anti-human BAFF-R blocking antibodies or the two combined. Suppression of $\operatorname{IgG}$ and $\operatorname{IgA}$ production is expressed as the percentage of reduction of the effect induced by TLR3 stimulation. * $\mathrm{p}<0.05, * * \mathrm{p}<0.01$ compared to PIC stimulated. 
(b) Modulation of AID mRNA expression in B cells co-cultured in transwell with RASF for $72 \mathrm{~h}$ after blockade of BAFFR and/or BCMA signalling. Results are expressed as mean \pm SEM of the mRNA fold increase calculated by setting the baseline expression as $1 .{ }^{*} \mathrm{p}<0.05$, $* * \mathrm{p}<0.01$ compared to PIC stimulated.

(c) Representative $1 \%$ agarose gel (inverted colour) of $\mathrm{I} \gamma$-C $\mu$ circular transcripts (CT, amplicon 502 bp, upper gel) expression in B cells co-cultured in transwell with RASF for 72h after blockade of BAFFR and/or BCMA signalling. Endogenous control, mammalian 18S (amplicon 187bp) is shown in the lower gel. 

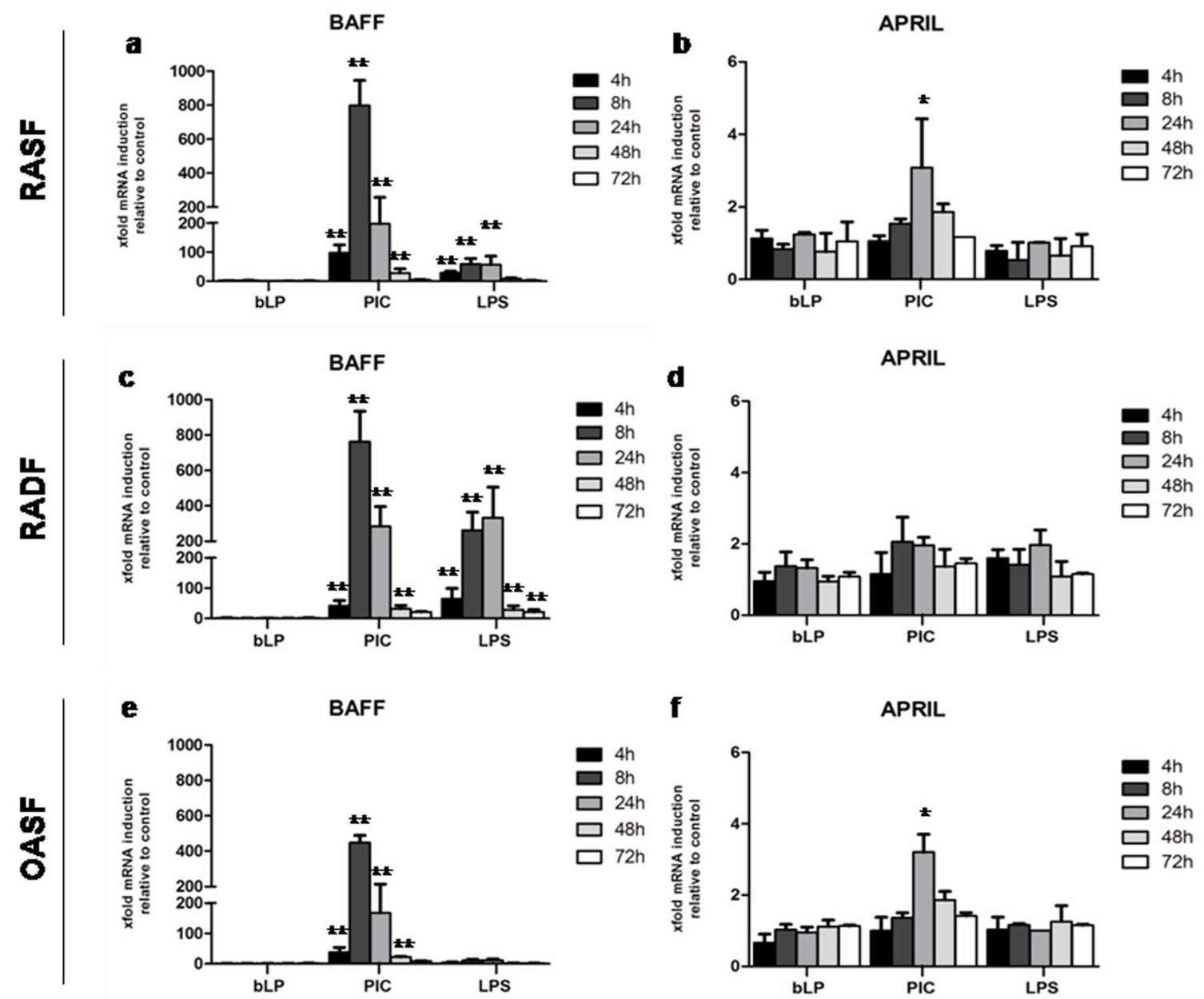

f

APRIL

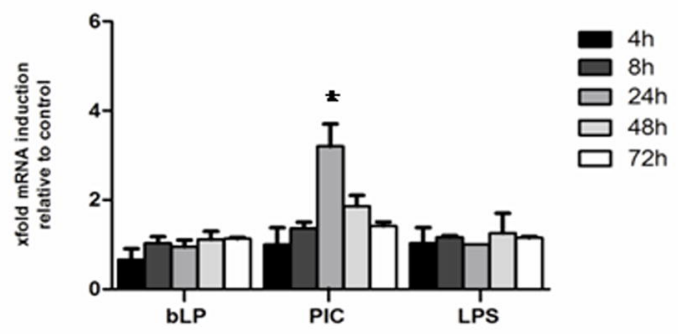




\section{Figure 2}

a

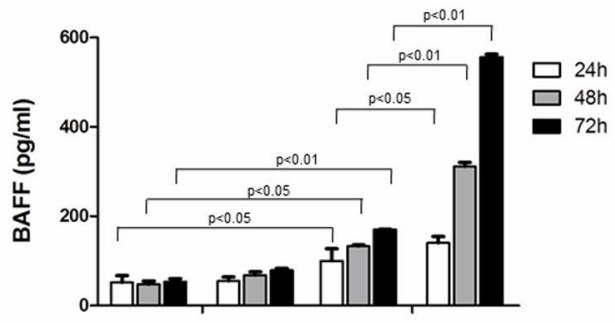

RADF

RASF

$\overline{\text { medium }} \overline{\mathrm{PIC}} \overline{\text { medium }} \overline{\mathrm{PIC}}$

medium only

DAPI BAFF
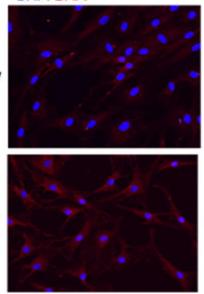

Isotype control

PIC
BAFF
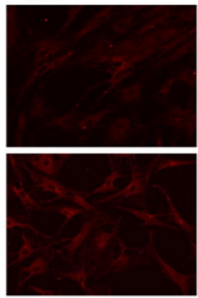

DAPI BAFF
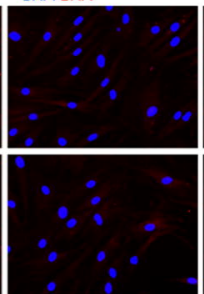

Isotype control

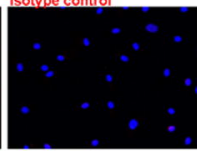

BAFF

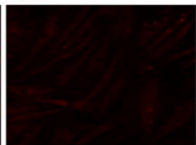

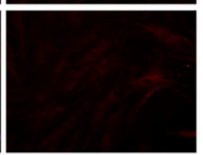
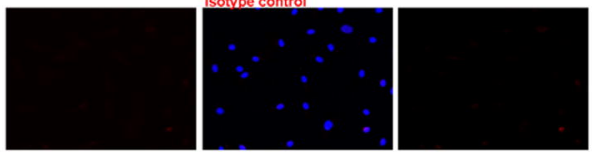

RADF 
Figure 3

A

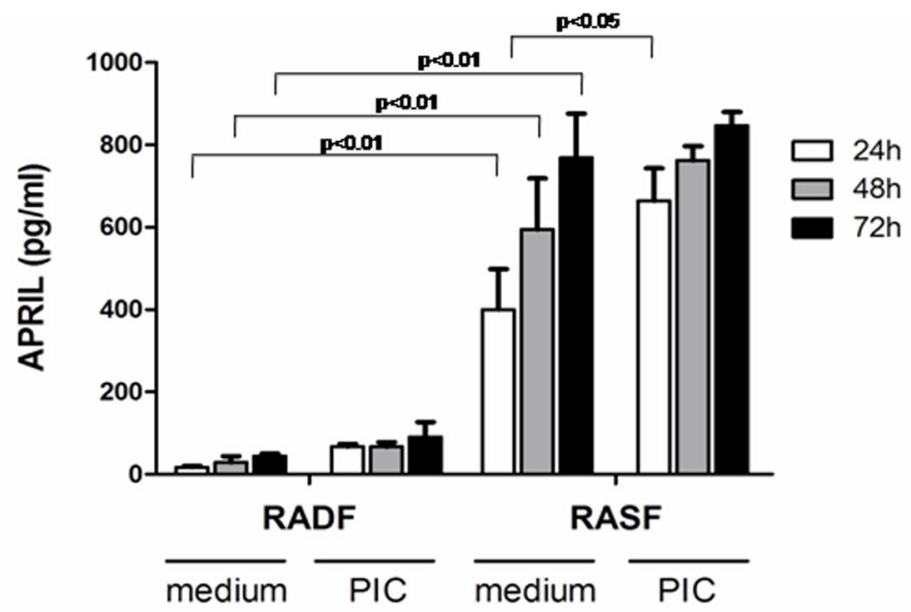

b

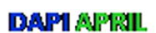

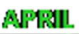

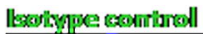
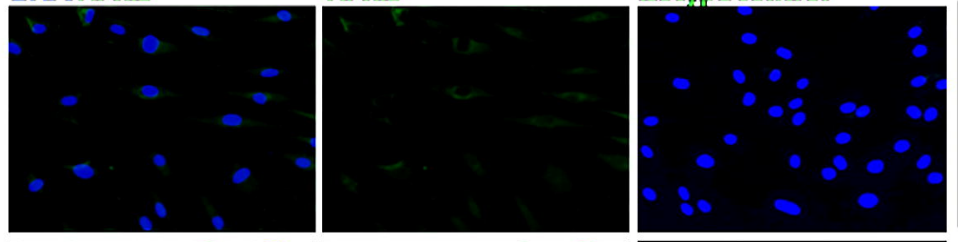

超韋章
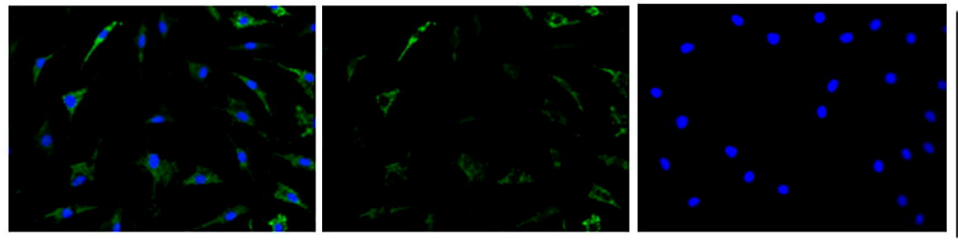

尽急 


\section{Figure 4}

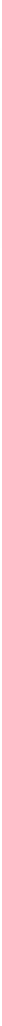


8

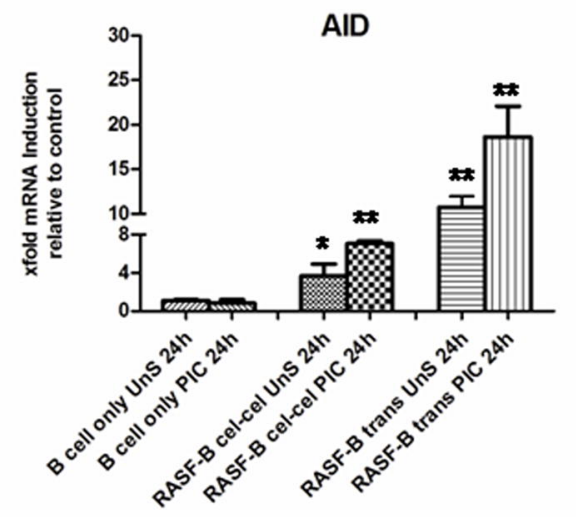

c

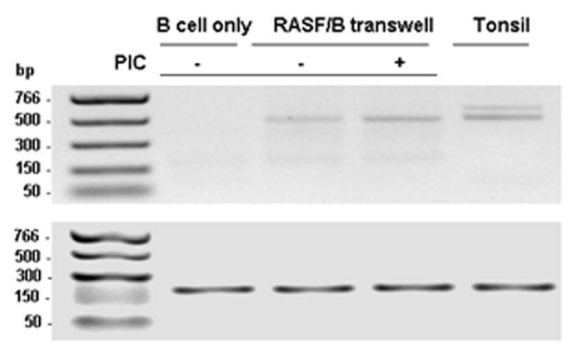

b

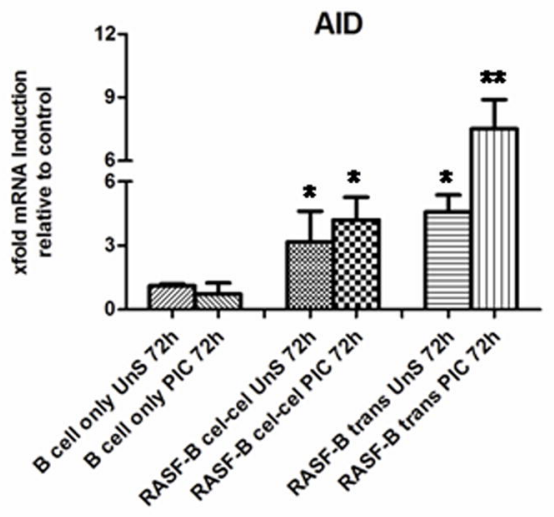

d

IgG/lgM ratio

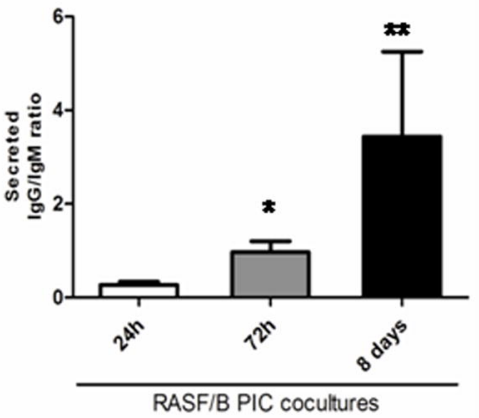




\section{Figure 6}

a
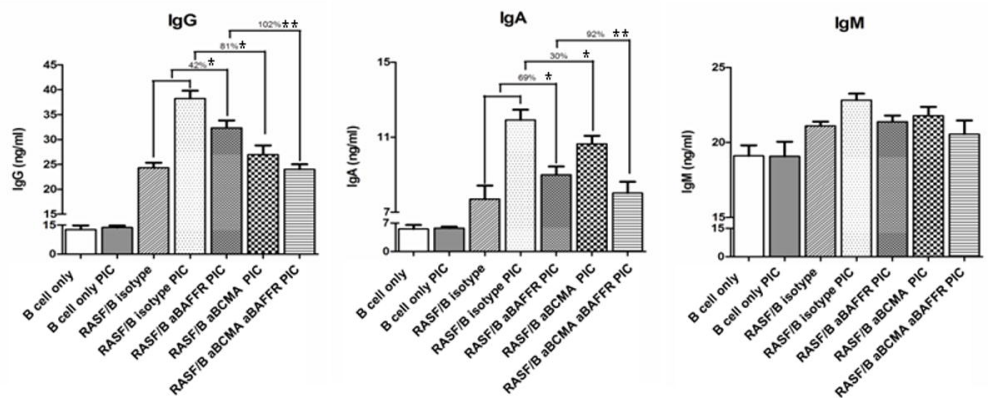

b
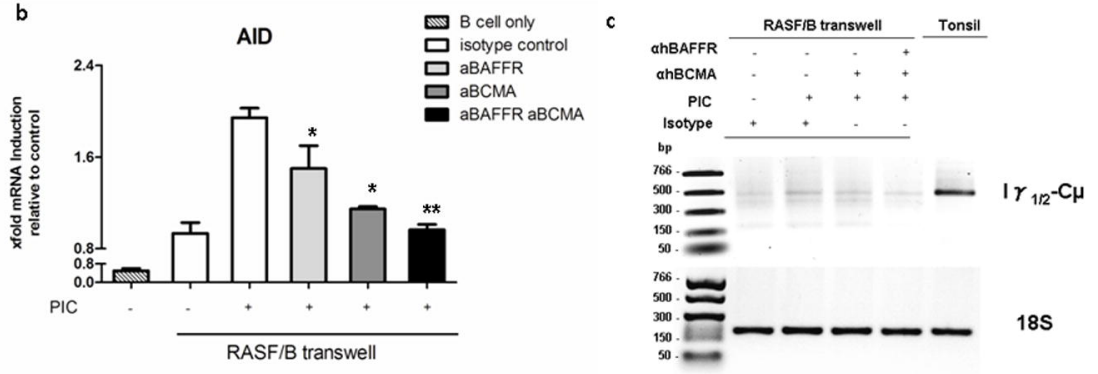